\title{
ESTUDO DE CRITÉRIOS LOCACIONAIS COM VISTAS AO ESTABELECIMENTO DE VANTAGEM COMPETITIVA
}

\author{
Carlos Navarro Fontanillas \\ UFRJ - Universidade Federal do Rio de Janeiro
}

\author{
Harvey Cosenza
}

UFRJ - Universidade Federal do Rio de Janeiro

Brasil

Luis Moura

UFRJ - Universidade Federal do Rio de Janeiro

Izabel Leonardo

UFRJ - Universidade Federal do Rio de Janeiro

\begin{abstract}
Martius Vicente Rodriguez y Rodriguez
UFF - Universidade Federal Fluminense

Brasil
\end{abstract}

11 Contextus

ISSN 1807-5908

Avaliação: Double Blind Review

Recebido em: 31.10.2017

$2^{\mathrm{a}}$ versão aceita para publicação em: 09.05.2018

DOI (artigo): doi.org/10.22409/1807-5908.i76a129 


\title{
Resumo
}

Atuando em um contexto empresarial, a localização tornou-se relevante para a competitividade das empresas. Este artigo objetivou identificar e caracterizar os critérios de localização que influenciam no estabelecimento de vantagem competitiva. O estudo se justifica e se faz relevante, pois pesquisas de localização sob a ótica das necessidades do usuário são escassos. Com a exploração dessa temática, pretendeu-se contribuir para aprimorar do planejamento de novos empreendimentos, objetivando uma melhor compreensão acerca da dinâmica da mutação das necessidades dos empreendedores.

De forma a garantir a racionalidade e consistência da metodologia, será utilizado o Método da Análise Hierárquica (MAH). Pretende-se assim utilizar a técnica para a solução de problemas de decisão multicritério, baseadas em decisões de valor.

Por fim, esta abordagem pretende oferecer subsídios aos critérios de locacionais com vistas a vantagem competitiva.

Palavras-Chave: Localização industrial; Método da Análise Hierárquica; Vantagem Competitiva.

\begin{abstract}
Acting in a business context, location has become relevant to the competitiveness of companies. This article aimed to identify and characterize the localization criteria that influence the establishment of competitive advantage. The study is justified and it becomes relevant, since searches of location from the point of view of user needs are scarce. With the exploration of this theme, it was intended to contribute to improve the planning of new ventures, aiming at a better understanding about the dynamics of the mutation of the entrepreneurs' needs.

To guarantee the rationality and consistency of the methodology, the Hierarchical Analysis Method (AHP) will be used. The aim is to use the technique for the solution of multicriteria decision problems, based on value decisions.

Finally, this approach intends to offer subsidies to locational criteria for competitive advantage.
\end{abstract}

Keywords: Industrial location; Analytic Hierarchy Process; Competitive Advantage. 


\section{Sustainable Business International Journal \\ SBIJ76 - MARÇO DE 2018 - ISSN 1807-5908}

\section{Introdução}

A localização é considerada um requisito utilitário determinante para um empreendimento e para seus potenciais investidores e clientes, não levando em conta somente o endereço do negócio, mas sim todo um conjunto de características, tais como: infraestrutura urbana (estacionamentos, restaurantes, agências bancárias, comércio varejista, hospitais, farmácia, lazer, entre outros), acessibilidade e serviços públicos (saneamento básico, transporte coletivo, cabos ópticos, rede de telefonia etc.).

De acordo com Guimarães (2000) algumas características não mensuráveis são especialmente representativas numa localização. Uma região pode não contar com sofisticados equipamentos urbanos e mesmo assim estar entre as preferidas dos usuários. Este é o caso de regiões que possuem grande diversidade de atrações culturais. Para serem tomadas as decisões corretas e precisas para tais objetivos, torna-se necessário um amplo planejamento estratégico.

O termo estratégia depende parcialmente do que se entende por organização. Se a mesma é formada por um grupo de porte e diversificada, sua estratégia a posicionará em um ambiente global, político, econômico e social e consistirá em decisões sobre os tipos de negócio o grupo quer conduzir, em que lugares do mundo deseja atuar, que negócios adquirir e de quais se desfazer, como alocar recursos em várias ações, como também nos projetos de produtos e/ou serviços.

O objetivo de projetar produtos e serviços consiste em satisfazer consumidores e atender suas necessidades (qualidade) e expectativas (marketing) atuais e futuras. Isto, por sua vez, melhora a competitividade da organização. Cosenza (2009) relata que pode-se observar que o projeto de produto e serviço, tem seu início com o consumidor e nele termina. 


\section{Sustainable Business International Journal}

Preliminarmente, a tarefa do marketing consiste em reunir informações acerca dos clientes (e, às vezes, de não clientes) para compreender e identificar suas necessidades alinhadas às premissas de qualidade e também para procurar possíveis oportunidades de mercado. Esta é uma tarefa complexa, que envolve a combinação de muitos aspectos diferentes dos objetivos de uma empresa. A especificação é então usada como entrada para a operação, que produz, fornece o produto ou serviços aos seus clientes. De acordo com Slack (2009), para obter o sucesso em tais objetivos e planejamentos, alguns aspectos de extrema importância devem ser destacados: localização, distribuição física e construção de vantagens competitivas, mediante a aplicação dos quatro tipos de desempenho: custo, qualidade, velocidade e inovação. Essas quatro dimensões, quando bem conduzidas, fornecem valor para o consumidor e vantagem competitiva para a organização.

Destacando-se nesses requisitos, enfatizamos os fatores locacionais, e é sobre ele que este trabalho pretende se deter e se propõe ao seguinte questionamento: A localização é um aspecto fundamental na construção da vantagem competitiva de uma organização?

Diante de tais critérios, utiliza-se a metodologia de estudo de caso em acordo com os conceitos de Vergara (2005), e a logística passa a adquirir importância estratégica, passando a planejar, implementar e controlar de maneira eficiente o fluxo de produtos. Há diversas técnicas usadas na localização de diferentes sítios e que, aparentemente, poderiam ser aplicados neste trabalho, tais como as relacionadas a seguir: teoria da localização, pesquisa operacional, método de análise hierárquica e lógica fuzzy.

\section{O Modelo Teórico}




\title{
Sustainable Business International Journal
}

O modelo utilizado neste paper é o Método de Análise Hierárquica (MAH), desenvolvido em meados da década de 70 por Thomas L. Saaty e destinado ao ambiente decisório multicritério. De acordo com Fontanillas (2014) no MAH a estruturação do problema é feita de forma intuitiva através de árvores hierárquicas possuindo um indicador de consistência reconhecível que consiste em avaliar o quanto o maior autovalor de uma matriz se afasta de sua ordem. Tal inferência é reconhecida pela equação do índice de consistência (IC):

$$
\mathrm{IC}=|(\lambda \max -\mathrm{N})| /(\mathrm{N}-1)
$$

Sendo $\mathrm{N}$ a ordem da matriz e $\lambda$ max indica o maior autovalor da matriz

Fontanillas (2014) discorre que o cálculo de autovalores envolve uma série de expressões e operações algébricas com matrizes. Não obstante, para o caso particular das matrizes de julgamentos de valores, Saaty (2000) expõe uma metodologia alternativa que permite o cálculo aproximado de $\lambda \max$, e se utiliza de uma escala principal assimilável por decisores. Apesar de o método estar sujeito a inversões de ordem, ou seja, a prioridade de uma alternativa A pode estar invertida em relação a uma alternativa $\mathrm{B}$ devido à inserção ou à retirada de uma terceira alternativa $\mathrm{C}$, o autor propõe o uso da razão de consistência ao final do processo para que seja avaliado se houve inconsistência ao processo. A razão da consistência é calculada pela fórmula:

\author{
$\mathrm{RC}=\mathrm{IC} / \mathrm{IR}$
}




\section{Sustainable Business \\ International Journal}

SBIJ76 - MARÇO DE 2018 - ISSN 1807-5908

em que IR é um índice de consistência desenvolvido em laboratório por meio de experimentos aleatórios que testa a consistência de um número extremamente grande de julgamentos. Entrementes, ele propõe a seguinte comparação:

Tabela 1

Índices de consistência randômicos

\begin{tabular}{|c|c|}
\hline Ordem da matriz & Valores de IR \\
\hline 3 & 0,58 \\
\hline 4 & 0,90 \\
\hline 5 & 1,12 \\
\hline 6 & 1,24 \\
\hline 7 & 1,32 \\
\hline 8 & 1,41 \\
\hline 9 & 1,45 \\
\hline
\end{tabular}

Nota. Fonte: Saaty (2000).

A comparação se dá por meio da razão entre o IC e o IR correspondente.

Esta abordagem processa uma grande vantagem devido a sua simplicidade e dessa forma foi escolhida para essa análise como representante de método de multicritério de apoio à decisão. Bentes, Carneiro, da Silva e Kimura (2011) relatam que certos problemas de tomada de decisão envolvem escolhas entre alternativas que são comparáveis usando vários critérios e como tal, podem apresentar algum grau de inconsistência. Não obstante, deriva-se um problema complexo através do uso da estrutura hierárquica até que a comparação entre dados possa ser feita de forma mais simples, facilitando assim sua compreensão e avaliação.

\subsection{Critérios de localização}


Os fatores relevantes para localizar instalações, apresentam-se como metodologia proposta para o presente artigo. Contudo, no intuito de validar a pesquisa, foram utilizados pesos relativos aos aspectos apresentados advindos da lógica fuzzy.

\subsubsection{Aspectos econômicos}

Nessa categoria foram analisadas seis ações de maior influência :

Custo: custo de implantação do empreendimento, manutenção, operadores e transporte de passageiros, empresas terceirizadas, impostos, benefícios sociais;

Distância: distância da instalação ao centro urbano e entroncamento rodoviário mais próximo;

Tempo: tempo de trânsito, viagem, transferência e freqüência do transporte urbano;

Segurança: no tráfego pelas condições das rodovias, transporte e terminais;

Impactos Ambientais: poluição sonora, poluição do ar, trepidação, poluição visual;

Impactos sócio-econômicos: viabilidade econômica das instalações e geração de empregos ao sistema.

\subsubsection{Aspectos operacionais}

Nessa categoria foram analisadas três ações de maior influência:

Confiabilidade: do serviço de: trânsito, infra-estrutura, capacidade e sua prestação;

Acessibilidade: congestionamentos nas proximidades entre a zona urbana e entroncamentos próximos;

Mobilidade: oferta de condições de acesso a empresa.

\subsubsection{Aspectos relativos à infraestrutura}




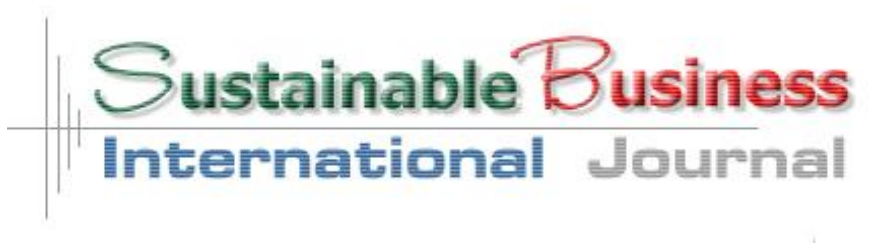

SBIJ76 - MARÇO DE 2018 - ISSN 1807-5908

Nessa categoria foram analisadas duas ações de maior influência:

a) Transporte Rodoviário de Passageiros: atendimento, conforto, segurança, pontualidade e custo do transporte;

b) Transporte Urbano e Metropolitano: integração intermodal, custo da viagem, atendimento, conforto e pontualidade.

\subsection{Alternativas de localização}

Inicialmente foi escolhida por meio de aspectos logísticos relevantes a forma de usarem as discussões para hierarquizar as ações. Foram consultados para fins da escolha e pesquisa dados do Ministério dos Transportes (ANTT) disponíveis no site do órgão. A escolha das alternativas deu-se em funções dos modais logísticos: portuários, terrestres (rodoviário e ferroviário), dutoviário e aéreo.

Através dos dados identificados conforme anexos, foram escolhidos os estados de Pernambuco, Rio de Janeiro e Rio Grande do Sul em função dos seguintes atributos:

Tabela 2 
Sustainable Business

International Journal

SBIJ76 - MARÇO DE 2018 - ISSN 1807-5908

Modais logísticos das cidades pesquisadas

\begin{tabular}{|c|c|c|c|}
\hline Atributos & Pernambuco (A) & Rio de Janeiro (B) & Rio Grande do Sul (C) \\
\hline Porto & $\begin{array}{l}2 \text { portos (Recife e } \\
\text { Suape) }\end{array}$ & $\begin{array}{l}2 \text { portos (Rio de Janeiro } \\
\text { Itaguaí ou Sepetiba) }\end{array}$ & $\begin{array}{c}2 \text { portos (Porto Alegre } \\
\text { e Rio Grande) }\end{array}$ \\
\hline Rodovia & BR 101 e BR 232 & $\begin{array}{c}\text { BR } 101, \text { BR } 116 \text { e BR } \\
040\end{array}$ & $\begin{array}{c}\text { BR 101, BR } 208 \text { e BR } \\
290\end{array}$ \\
\hline Ferrovia & 1 ferrovia & 1 ferrovia & 1 ferrovia \\
\hline Gasoduto & 1 gasoduto & 1 gasoduto & 1 gasoduto \\
\hline Aéreo & $\begin{array}{l}1 \text { aeroporto } \\
\text { internacional }\end{array}$ & $\begin{array}{l}1 \text { aeroporto } \\
\text { internacional }\end{array}$ & $\begin{array}{l}1 \text { aeroporto } \\
\text { internacional }\end{array}$ \\
\hline
\end{tabular}

Nota. Elaborado pelos autores.

Acrescenta-se à pesquisa, que todos os dados foram coletados e analisados dando-se um grau de importância adicional ao fato do Rio de Janeiro possuir um porto com dimensões de calado superior a 15 metros, item fundamental para qualquer pretensão de escoamento de insumos ou produtos acabados na cadeia logística, e que, pela falta de estrutura na geração de energia nacional, o gasoduto faz-se necessário pelo seu uso na geração energética. A proximidade de grandes centros nos remete à convicção no sentido da rapidez logística, principalmente nos casos oriundos e relacionados a itens de menor escala, para escoamento rodoviário e aeroviário salvo melhor juízo. 


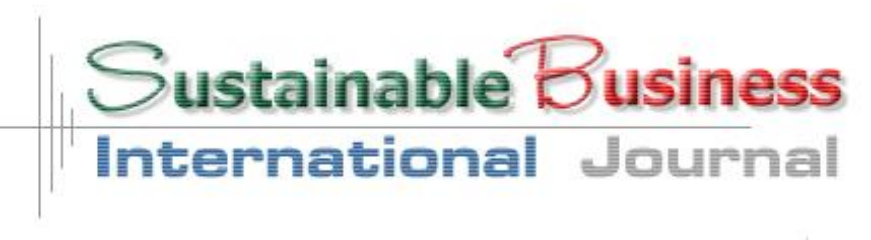

SBIJ76 - MARÇO DE 2018 - ISSN 1807-5908

Nesse sentido, o Método da Pontuação Ponderada envolve a identificação de critérios que podem ser usados para avaliar diversas localizações. Slack (2009) insere-se uma definição da importância relativa a cada critério e a atribuição de fatores de ponderação (pesos) para cada um deles. Posteriormente é avaliada cada localização segundo cada critério. A totalidade dos pesos atribui-se 100.

Entrementes, para uma indústria se localizar, ponderam-se arbitrariamente numa escala de 0 a 100 os seguintes aspectos:

Tabela 3

\section{Ponderação dos atributos}

\begin{tabular}{|c|c|c|c|c|}
\cline { 3 - 5 } \multicolumn{2}{c}{} & \multicolumn{3}{c|}{ PONTUAÇÃO } \\
\cline { 3 - 5 } \multicolumn{2}{c}{} & \multicolumn{3}{c|}{ PONDERAÇÃO DE LOCAIS } \\
\cline { 3 - 5 } \multicolumn{2}{c}{} & A & B & C \\
\hline Critérios & Importância & Pernambuco & Rio de Janeiro & Rio Grande do Sul \\
\hline Potencial de calado & 4 & 60 & 90 & 50 \\
\hline Escoamento por ferrovias & 2 & 80 & 50 & 40 \\
\hline Escoamento por rodovias & 1 & 60 & 80 & 70 \\
\hline Proximidade de aeroporto & 1 & 90 & 30 & 70 \\
\hline Gasoduto & 3 & 70 & 70 & 70 \\
\hline Proximidade dos centros & 3 & 60 & 90 & 860 \\
\hline Total & - & 880 & 1050 & 90 \\
\hline
\end{tabular}

Nota. Fonte: Elaborado pelos autores. 


\section{Sustainable Business International Journal}

Pela metodologia tem-se que o Rio de Janeiro possuiu uma pontuação maior que as demais e seria a melhor opção dentre as três apresentadas. Pernambuco e Rio Grande do Sul ficaram muito próximos, mas aquém do Rio de Janeiro, que neste caso seria a escolhida.

\section{Metodologia}

Para o experimento utilizando o método da Análise Hierárquica (MAH), uma representação foi estruturada hierarquicamente conforme ilustra a figura 1:

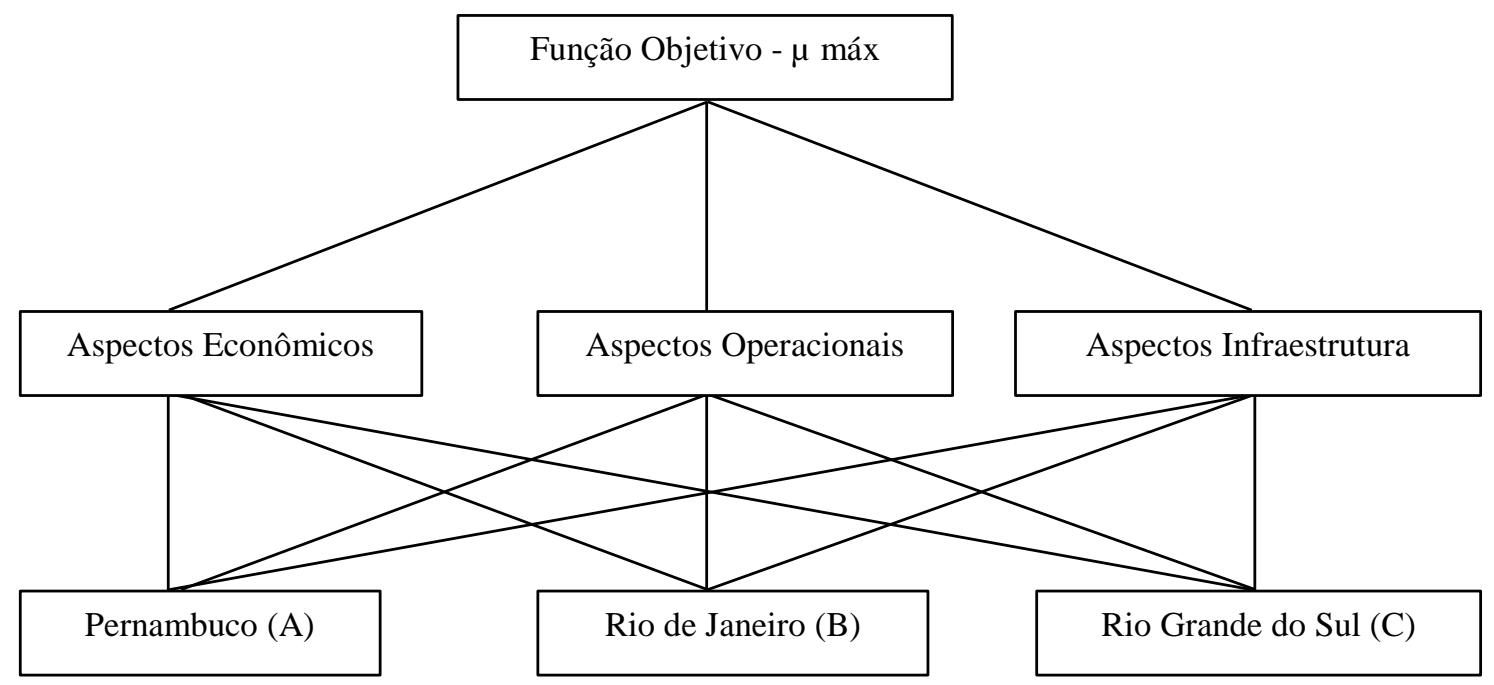

Figura 1. Hierarquia da Análise

Na Figura 1, elaborada pelos autores, demonstra-se a função objetivo, os critérios e alternativas a serem analisados.

O modelo buscou estabelecer uma escala cardinal e ordinal dessas variáveis. A partir de subjetividade dos decisores, gerando uma escala de pesos relativos advindos da lógica fuzzy como visto: 


\section{Sustainable Business International Journal}

SBIJ76 - MARÇO DE 2018 - ISSN 1807-5908

Tabela 4

\section{Pesos dos critérios}

\begin{tabular}{|c|c|}
\hline Critérios Atribuídos & Peso do critério \\
\hline Aspectos econômicos & 5 \\
\hline Aspectos operacionais & 3 \\
\hline Aspectos de infraestrutura & 2 \\
\hline
\end{tabular}

Nota. Fonte: Elaborada pelos autores.

Tabela 5

Demonstração da avaliação das alternativas em relação aos critérios, em uma tabela separada

\begin{tabular}{|c|c|c|c|}
\hline Alternativas & Critério & Critério & Critério \\
\hline Pernambuco & Aspectos econômicos & Aspectos operacionais & Aspectos infraestrutura \\
\hline Rio de Janeiro & Aspectos econômicos & Aspectos operacionais & Aspectos infraestrutura \\
\hline Rio Grande do Sul & Aspectos econômicos & Aspectos operacionais & Aspectos infraestrutura \\
\hline
\end{tabular}

Nota. Fonte: Elaborada pelos autores.

Baseando-se na hierarquia, como mostra a tabela, e de acordo com resultados obtidos da convicção do observador, realiza-se uma matriz de relacionamentos paritários. Essa matriz refere-se ao critério aspectos econômicos, e informa, com relação a esse critério, o quanto uma determinada alternativa é superior à outra. 


\section{Sustainable Business \\ International Journal}

SBIJ76 - MARÇO DE 2018 - ISSN 1807-5908

Tabela 6

Relacionamento paritário do fator econômico

\begin{tabular}{|c|c|c|c|}
\cline { 2 - 4 } \multicolumn{1}{c|}{} & Pernambuco & Rio de Janeiro & Rio Grande do Sul \\
\hline Pernambuco & 1 & $1 / 7$ & $1 / 3$ \\
\hline Rio de Janeiro & 7 & 1 & 5 \\
\hline Rio Grande do Sul & 3 & $1 / 5$ & 1 \\
\hline
\end{tabular}

Nota. Fonte: Elaborada pelos autores.

Tabela 7

Matriz com as colunas normalizadas

\begin{tabular}{|c|c|c|c|}
\hline Alternativas/Alternativas & A & B & C \\
\hline A & 0,092 & 0,106 & 0,054 \\
\hline B & 0,636 & 0,745 & 0,789 \\
\hline C & 0,272 & 0,149 & 0,157 \\
\hline
\end{tabular}

Nota. Fonte: Elaborada pelos autores.

Tabela 8

Soma das linhas da matriz

\begin{tabular}{|c|c|c|}
\hline Pernambuco & Rio de Janeiro & Rio Grande do Sul \\
\hline 0,084 & 0,723 & 0,193 \\
\hline
\end{tabular}

Nota. Fonte: Elaborada pelos autores. Vetor de prioridades, evidenciando a escala de preferências das alternativas.

Cruz (2007) declara que é necessário verificar se os resultados estão coerentes com o pressuposto de racionalidade. Para tal, calcula-se o autovalor principal da matriz, comparando com o índice de consistência obtido com o índice randômico das matrizes recíprocas de ordem três para se obter uma razão de consistência. 


\section{Sustainable Business International Journal}

SBIJ76 - MARÇO DE 2018 - ISSN 1807-5908

Tabela 9

Multiplicação a matriz original pelo vetor

\begin{tabular}{|c|c|c|}
\hline Pernambuco & Rio de Janeiro & Rio Grande do Sul \\
\hline 0,251 & 2,275 & 0,590 \\
\hline
\end{tabular}

Nota. Fonte: Elaborada pelos autores.

Tabela 10

Divisão dos valores do vetor pelos valores do autovetor de prioridades

\begin{tabular}{|c|c|c|}
\hline Pernambuco & Rio de Janeiro & Rio Grande do Sul \\
\hline 2,988 & 3,145 & 3,061 \\
\hline
\end{tabular}

Nota. Fonte: Elaborada pelos autores.

Somando-se estes componentes e tomando sua média tem-se: 3,06

Logo, pela metodologia com o autovalor máximo desta matriz obtêm-se o índice de consistência (IC).

I.C. $=\left(\lambda_{\text {máx }}-\mathrm{n}\right) /(\mathrm{n}-1)=(3,06-3) / 2=0,03$

Dividindo-se o resultado pelo índice randômico para matrizes de ordem três, com valor de 0,58 encontra-se uma razão de consistência ( $\mathrm{RC}$ ) de 0,05 confirmando a racionalidade da escolha. 


\section{Sustainable Business International Journal}

Tabela 11

Procedimento para o critério aspecto operacional

\begin{tabular}{|c|c|c|c|}
\hline & Pernambuco & Rio de Janeiro & Rio Grande do Sul \\
\hline Pernambuco & 1 & $1 / 3$ & 3 \\
\hline Rio de Janeiro & 3 & 1 & 7 \\
\hline Rio Grande do Sul & $1 / 3$ & $1 / 7$ & 1 \\
\hline
\end{tabular}

Nota. Fonte: Elaborada pelos autores.

Tabela 12

Divisão dos valores do vetor pelos valores do autovetor de prioridades

\begin{tabular}{|c|c|c|}
\hline Pernambuco & Rio de Janeiro & Rio Grande do Sul \\
\hline 4,210 & 3,518 & 1,725 \\
\hline
\end{tabular}

Nota. Fonte: Elaborada pelos autores. Com essa matriz a razão de consistência tem valor igual a zero (0) que está de acordo com a hipótese de consistência perfeita.

Tabela 13

\section{Metodologia para o critério aspectos de infraestrutura}

\begin{tabular}{|c|c|c|c|}
\cline { 2 - 4 } \multicolumn{1}{c|}{} & Pernambuco & Rio de Janeiro & Rio Grande do Sul \\
\hline Pernambuco & 1 & $1 / 3$ & 2 \\
\hline Rio de Janeiro & 3 & 1 & 1 \\
\hline Rio Grande do Sul & $1 / 2$ & $1 / 5$ & 5 \\
\hline
\end{tabular}

Nota. Fonte: Elaborada pelos autores. 


\section{Sustainable Business International Journal}

SBIJ76 - MARÇO DE 2018 - ISSN 1807-5908

Tabela 14

Divisão dos valores do vetor pelos valores do autovetor de prioridades

\begin{tabular}{|c|c|c|}
\hline Pernambuco & Rio de Janeiro & Rio Grande do Sul \\
\hline 2,999 & 2,998 & 3,003 \\
\hline
\end{tabular}

Nota. Fonte: Elaborada pelos autores. Nessa matriz a razão de consistência tem valor igual a 0,07 evidenciando que a ordenação é compatível com a racionalidade.

O próximo passo consiste na elaboração de uma matriz de relacionamentos entre os aspectos envolvidos, ou seja, sua hierarquia. Como será mostrado na tabela que se segue, adotou-se uma relação de pesos diretos de acordo com a pesquisa delineada, respaldada nos conceitos da lógica nebulosa advindos de Shaw (2001).

\section{Tabela 15}

\section{Matriz de relacionamento dos aspectos}

\begin{tabular}{|c|c|c|c|}
\cline { 2 - 4 } \multicolumn{1}{c|}{} & Pernambuco & Rio de Janeiro & Rio Grande do Sul \\
\hline Pernambuco & 1 & 6 & 4 \\
\hline Rio de Janeiro & $1 / 6$ & 1 & $1 / 3$ \\
\hline Rio Grande do Sul & $1 / 4$ & 3 & 1 \\
\hline
\end{tabular}

Nota. Fonte: Elaborada pelos autores.

A razão de consistência encontrada tem valor igual a 0,04 que evidencia uma racionalidade coerente com a escolha feita para obtenção de vantagens competitivas.

A seguir demonstra-se o resultado consistente dos critérios em relação a todas as alternativas. 


\section{Sustainable Business International Journal}

SBIJ76 - MARÇO DE 2018 - ISSN 1807-5908

Tabela 16

\section{Compilação dos resultados critérios $x$ alternativas}

\begin{tabular}{|c|c|c|c|}
\cline { 2 - 4 } \multicolumn{1}{c|}{} & $C_{1}$ & $C_{2}$ & $C_{3}$ \\
\hline$I C$ & 0,03 & 0,00 & 0,04 \\
\hline$R C$ & 0,05 & 0,00 & 0,07 \\
\hline
\end{tabular}

Nota. Fonte: Elaborada pelos autores.

Como todas as consistências estão abaixo do valor máximo tolerado, pode-se afirmar que o modelo é consistente. Na existência de inconsistências para alguns critérios, o decisor deverá reavaliar as hierarquias e encontrar o ponto de inconsistência.

Ressalta-se que o resultado expressa a melhor alternativa entre as selecionadas para o resultado final que nesse contexto refere-se à localização de uma indústria com fins de obtenção de vantagem competitiva. Os testes de consistência indicaram que o modelo adequou-se aos pesos atribuídos nos critérios e alternativas.

Finalizando, consolidam-se os resultados encontrados dos autovetores de preferência com os pesos relativos a cada critério, obtendo-se:

Tabela 17

\section{Resultados consolidados}

\begin{tabular}{|c|c|c|}
\hline Pernambuco & Rio de Janeiro & Rio Grande do Sul \\
\hline 0,151 & 0,693 & 0,147 \\
\hline
\end{tabular}

Nota. Fonte: Elaborada pelos autores.

Dessa forma, os valores relativos passam a ser o resultado definitivo do experimento, no tocante à ordenação das preferências de seus aspectos, e aderentes ao Método de Pontuação Ponderada proposto por Slack (2009). 


\section{Sustainable Business International Journal}

SBIJ76 - MARÇO DE 2018 - ISSN 1807-5908

\section{Conclusões}

Neste artigo analisou-se primeiramente o conceito de localização para em um segundo momento analisar os critérios que integram o composto mercadológico. A seguir, demonstrouse que a estratégia competitiva dos negócios configura-se a partir da relação entre qualidade da localização e infraestrutura praticada. Esta composição desenha a estratégia de inserção de mercado do produto.

Do exposto, conclui-se que os aspectos que irão conferir segurança ao investimento com a instalação de uma empresa encontram-se relacionados a seu desempenho no período de exploração, assegurando um fluxo de renda atrativo. Ou seja, o tempo necessário para o empresário reaver o montante imobilizado, bem como o retorno do investimento, depende de seu desempenho mercadológico, que, por sua vez, depende das adaptações de seus atributos à necessidade dos clientes.

Outro fato que merece ressalva tem relaçãoo à estruturação dos critérios e seus atributos. Usou-se o conceito da lógica nebulosa com a estruturação do Método de Análise Hierárquica.

De tal maneira, o uso da abordagem de Saaty apoiada pela lógica fuzzy, passa a ser uma metodologia indicada para balizar os pressupostos em tomada de decisão de uma forma consistente, pois encontra validação acadêmica através da pesquisa bibliográfica sobre o assunto.

Não obstante, corroboram-se conclusões com as intenções do trabalho. O objetivo foi apresentar um método (multicritério e multidecisor) aplicado a um empreendimento industrial para obtenção de vantagem competitiva. Entrementes, foi alcançado, uma vez que as metodologias clássicas foram apresentadas, definidas e aplicadas à proposta. 


\section{Sustainable Business International Journal}

SBIJ76 - MARÇO DE 2018 - ISSN 1807-5908

Nesse sentido, entende-se que o uso das ferramentas de suporte a decisão está adequadamente contextualizado com a doutrina dos fatores de localização a fim de se obter vantagem competitiva.

\section{Referências}

Agência Nacional de Transportes Terrestres. (2008). Mapas. Recuperado de http://www.antt.gov.br/mapas/mapas.asp

BALLOU, R H. (2006). Gerenciamento da Cadeia de Suprimentos/Logística Empresarial. São Paulo: Bookman, 152.

BENTES, Alexandre Veronese, CARNEIRO, Jorge, SILVA, Jorge Ferreira da, \& KIMURA, Herbert. (2011). Multidimensional assessment of organizational performance: integrating BSC and AHP. Journal of Business Research, (65), 1790-1799.

BIAN, K.A. (2011). Application of Fuzzy AHP and ELECTRE to China Dry Port Location Selection. The Asian Journal of Shipping and Logistics, 27(2), 331-354.

BUYUKOZKAN, Gulçin, FEYZIOGLU, Orhan, \& NEBOL, Erdal. (2008). Selection of the strategic alliance partner in logistics value chain. International Journal of Production Economics, 113, 148-158.

NUNES, Carolina, RIBEIRO,Uila, MURTA,Aurélio, RODRIGUEZ,Martius, SILVA, Camilo. (2016). Uma Nova Abordagem da Área de Suprimentos. Sustainable Business International Journal, dezembro 2016.

CHOUDHARY, Devendra, \& SHANKAR, Ravi. (2012). An STEEP-fuzzy AHP-TOPSIS framework for evaluation and selection of thermal power plant location: A case study from India.Energy, 42, 510-521. 
COSENZA, Carlos Alberto Nunes, LIMA, Fernando Rodrigues, \& NEVES, César das. (2009). Modelos de Localização em Engenharia Urbana. Simpósio de Pós-Graduação em Engenharia Urbana.1-15.Recuperado de http://www.dec.uem.br/eventos/ii_simpgeu/arquivos/Trabalhos/131.pdf COVA, CJG. (2000). Decisão Orçamentária Pública: Uma Proposta de Metodologia de Tomada de Decisão e Avaliação dos Resultados (Tese de doutorado).Universidade Federal do Rio de Janeiro, Rio de Janeiro, RJ, Brasil, 53.

CRUZ, EP. (2007). Modelo multicritério e multidecisor para priorização de cadeias em Ciência, Tecnologia e Inovação: um estudo no Fórum de Competitividade da Cadeia Produtiva da Indústria Farmacêutica (Tese de doutorado). Universidade Federal do Rio de Janeiro, Rio de Janeiro, RJ, Brasil, 89.

FONTANILLAS, Carlos, CRUZ, Eduardo, \& BARRETO, Cesar. (2014). O processo decisório nas organizações (1a ed.). Curitiba: Intersaberes, 242.

FONTANILLAS, Carlos, CODEÇO, Monica, GARCIA, Sergio, LEONARDO, Izabel. (2015) O gerenciamento de um prestador de serviços logísticos na cadeia de suprimentos da saúde pública brasileira: um estudo de caso. Revista Sustainable Business International Journal, 48. GARTNER, Ivan Ricardo, ROCHA, Carlos Henrique, \& GRANEMANN, Sérgio Ronaldo. (2012). Modelagem multicriterial aplicada a problemas de regulação em áreas portuárias privatizadas. Revista de Administração Contemporânea, 16 (4),493-517. Recuperado de http://www.scielo.br/scielo.php?script=sci_arttext\&pid=S1415- 


\section{Sustainable Business \\ International Journal}

SBIJ76 - MARÇO DE 2018 - ISSN 1807-5908

GUARNIERI, Patricia. (2015). Síntese dos principais critérios, métodos e subproblemas da seleção de fornecedores multicritério. Revista de Administração Contemporânea, 19(1), 1-25. Recuperado de $\quad$ http://www.scielo.br/scielo.php?script=sci_arttext\&pid=S1415$\underline{6552015000100003 \& \operatorname{lng}=p t \& n r m=i s o \& t \operatorname{lng}=p t}$

HONG, Liu, \& XIAOHUA, Zhang. (2011). Study on location selection of multi-objective emergency logistics center based on AHP. Procedia Engineering, 15, 2128-2132.

KENGPOL, Athakorn. (2008). Design of a decision support system to evaluate logistics distribution network in Greater Mekong Subregion Countries. International Journal of Production Economics, 115, 388-399.

KUO, R.J., CHI, S.C., \& KAO, S.S. (1999). A Decision Support System for Locating Convenience Store through Fuzzy AHP. Computers and Industrial Engineering, 37, 323-326. MOORI, Roberto Giro, \& RIQUETTI, Alessandro. (2014). Estação de transbordo de cargas como mediador da logística de fertilizantes. Revista de Administração Contemporânea, 18 (6), 748-771.Recuperado de http://www.scielo.br/scielo.php?script=sci_arttext\&pid=S1415$\underline{65552014000600748 \& \operatorname{lng}=\mathrm{pt} \& \mathrm{nrm}=\mathrm{i} \text { so\&tlng }=\mathrm{pt}}$

MAIRI, C.G., MIRANDA,M.C., CARRARO,I.R.,FURLAN,J. (2016). Planejamento Estratégico na Logística: etapas e estratégias para a implementação. Revista Sustainable Business International Journal, 67.

MOSADEGHI, Razieh, WARNKEN, Jan, TOMLINSON, Rodger, \& MIRFENDERESK, Hamid. (2015). Comparison of Fuzzy-AHP and AHP in spatial multi-criteria decision making model for urban land-use planning. Computers,Environment and Urban Systems, 49, 54-65. 


\section{Sustainable Business International Journal \\ SBIJ76 - MARÇO DE 2018 - ISSN 1807-5908}

OZGEN, Dogan, \& GULSUN, Bahadir. (2014). Combining possibilistic linear programming and fyzzy AHP for solving the multi-objective capacitated multi-facility location problem. Information Sciences Journal, 268, 185-201.

PEDRYCZ, Witold, \& SONG, Mingli. (2014). A granulation of linguistic information in AHP decision-making problems. Information Fusion, 17, 93-101.

PRAHALAD, C.K., \& HAMEL, G. (1990). The core competence of the Corporation. Harvard Business Review, 79-91.

SAATY, TL. (2000). Método de Analise Hierárquica. São Paulo: Makron Books, 95.

SAATY, TL. (2006). Fundamentals of Decision Making and Priority Theory with the Analitic Hierarchy Process (2a ed.). Pensilvânia: RWS Publications, 70.

Serviço Brasileiro de Apoio às Micro e Pequenas Empresas. Localização e estrutura. (2007). Recuperado de http://www.sebrae.com.br/br/parasuaempresa/localizacaoeestrutura.asp SIMON, Alexandre Tadeu, SERIO, Luiz Carlos Di, PIRES, Silvio Roberto Ignacio, \& MARTINS, Guilherme Silveira. (2015). Evaluating Supply Chain Management: A methodology based on a theoretical model. Revista de Administração Contemporânea, 19(1), 26-44. Recuperado de http://www.scielo.br/scielo.php?script=sci_arttext\&pid=S1415$\underline{65552015000100004 \& \operatorname{lng}=\mathrm{pt} \& \mathrm{nrm}=\mathrm{iso} \& \operatorname{tng}=\mathrm{en}}$

SHAW, IS, \& SIMÕES, MG. (2001). Controle e Modelagem Fuzzy. São Paulo: Edgard Blucher, 88 .

SLACK, Nigel, CHAMBERS, Stuart, \& JOHNSTON, Robert. (2009). Administração da Produção (3a ed.). São Paulo: Atlas, 185. 


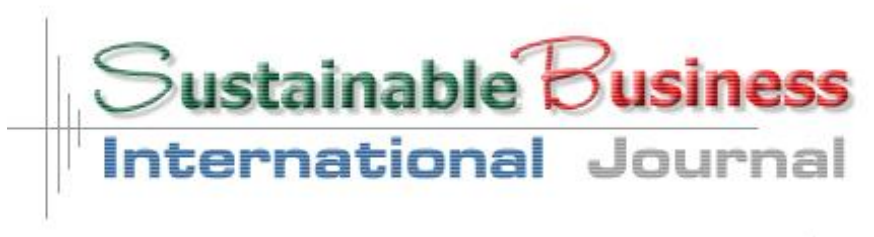

SBIJ76 - MARÇO DE 2018 - ISSN 1807-5908

VERGARA, Sylvia Constant. (2005). Projetos e Relatórios de Pesquisa em Administração. São Paulo: Atlas, 93.

WANG, Ying, JUNG, Kyung-Ae, YEO, Gi-Tae, \& CHOU, Chien-Chang. (2014). Selecting a cruise port of call location using the fuzzy-AHP method: A case study in East Asia. Tourism Management, 42, 262-270. 